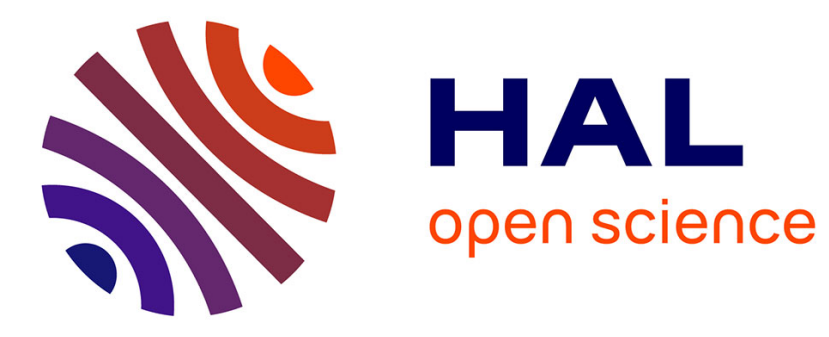

\title{
Guided Tearing: the ruler test
}

\author{
Alejandro Ibarra, Juan-Francisco Fuentealba, José Bico, Benoît Roman, \\ Francisco Melo
}

\section{To cite this version:}

Alejandro Ibarra, Juan-Francisco Fuentealba, José Bico, Benoît Roman, Francisco Melo. Guided Tearing: the ruler test. Physical Review Materials, 2021, 5 (2), 10.1103/PhysRevMaterials.5.025601 . hal-03402424

\section{HAL Id: hal-03402424 https://hal.sorbonne-universite.fr/hal-03402424}

Submitted on 25 Oct 2021

HAL is a multi-disciplinary open access archive for the deposit and dissemination of scientific research documents, whether they are published or not. The documents may come from teaching and research institutions in France or abroad, or from public or private research centers.
L'archive ouverte pluridisciplinaire HAL, est destinée au dépôt et à la diffusion de documents scientifiques de niveau recherche, publiés ou non, émanant des établissements d'enseignement et de recherche français ou étrangers, des laboratoires publics ou privés. 


\title{
Guided Tearing: the ruler test
}

\author{
Alejandro Ibarra, ${ }^{1}$ Juan-Francisco Fuentealba, ${ }^{1}$ José Bico, ${ }^{2}$ Benoît Roman, ${ }^{2}$ and Francisco Melo ${ }^{1}$ \\ ${ }^{1}$ Departamento de Física Universidad de Santiago de Chile, \\ Avenida Ecuador 3493, 9170124 Estación Central, Santiago, Chile \\ Center for Soft Matter Research, SMAT-C, Avenida Bernardo O'Higgins 3363, Estación Central, Santiago, Chile \\ ${ }^{2}$ PMMH, CNRS, ESPCI Paris, Université PSL, Sorbonne Université, Université de Paris, F-75005, Paris, France
}

(Dated: January 23, 2021)

\begin{abstract}
The physical rules governing the tearing of a packaging or of a piece of paper are not completely elucidated despite being common phenomena in daily life. Here, we investigate how the presence of a straight rigid object, a ruler, guides the fracture of a thin sheet within a wide range of pulling directions. In the case of thin isotropic brittle sheets, a simple geometrical analysis shows that fracture follows the direction of a tearing vector, which differs significantly from the pulling direction. In addition to geometry, bending energy or material anisotropy has to be implemented in the case of thicker or anisotropic sheets, to predict the direction of propagation. A generalization of the Wulff's type construction introduced by Takei et al. 1] accounts successfully for our experimental results.
\end{abstract}

\section{INTRODUCTION}

Opening a packaging is generally a frustrating experience as the fracture propagates along an undesired path. Pulling away a strip of adhesive tape for instance leads to a pointy shape, which is very inefficient in terms of unpacking 2-4. In ductile sheets, plasticity may lead to complex shapes as torn edges are irreversibly stretched, inducing longitudinal out-of-plane oscillations [5] - In brittle thin sheets, controlled model experiments show that tearing crack paths can be surprisingly regular. Logarithmic spirals [8, 9], oscillating [10 13], converging [14] or hyperbolic [15, 16] paths have for instance been observed and described [17].

Because of the relative simplicity of experimental setups, and the remarquable reproducibility of the crack path, fracture in thin sheets stands as a good system to probe fundamental questions on the selection of the direction of propagation. In isotropic continuous propagation, it is accepted that fracture follows the direction that maximizes the energy release rate [18, 19]. However, in the more general case of an anisotropic material, the question of the direction of propagation is still debated. Most manufactured films are anisotropic, which strongly influences their tearing properties [20]. Here we will further test a natural extension of the standard maximum energy release rate criterion for anisotropic sheets [1, 15].

In practical tearing applications, we often require a fracture to propagate in a given direction. A common solution consists in guiding tearing with a ruler. Although tearing the free "leg" of a notched sheet of paper is an intuitive operation, what are the physical bases of this process? What is the force required to tear the sheet? Does the crack always follow the ruler? What is the most efficient way to control tearing in the case of anisotropy of the fracture energy? We propose to address these questions through a model experiment where the material direction of the specimen and the pulling direction of the free leg can be controlled independently.
This study follows the same framework developed for a trouser test configuration [15 motivated by a seminal article by O'Keefe [21]. However, the presence of the ruler modifies significantly the geometry since the sheet laying under the ruler is forced to remain flat.

We first present the experimental setup and the geometrical ingredients of the problem. We then predict through geometrical arguments the direction of propagation of a crack and the required pulling force for an isotropic sheet. Although this geometrical description captures most of the fracture features, we find that the crack path deviates from the prediction, and in particular may leave the ruler away in some cases. We suggest that this deviation is due to anisotropy of the material, and to the neglected the elastic bending energy. We finally provide a more general framework which accounts for these additional physical effects.

\section{GEOMETRY OF THE EXPERIMENT}

The experiment aiming to mimic the common technique of tearing guided with a ruler is as follows. We start by cutting a rectangular sample in a sheet of polypropylene, as sketched in Fig 1 1a. Because materials are generally anisotropic, we will keep track of all orientations with respect to a fixed "material axis" (MA). A notch is performed and a ruler is fixed above half of the sample (above flap B). We note $\alpha_{r}$ the direction of the ruler (and notch) with respect to the Material Axis. The film of polypropylene selected for this study has a low anisotropy arising from the elongation imposed to the film during its manufacture. It will be demonstrated later that the material axis is one of the symmetry axis of the fracture energy of the film. The ruler is tightly pressed against the film in such a way that no slip boundary conditions are imposed along the entire ruler width and the sample. A flexible wire is attached to an arbitrary "pulling point" on 
flap A. This wire is pulled along a constant direction, defined by the unit vector $\hat{\tau}$, and at constant speed, thanks to a feedback controlled stage and the pulling force $F$ is monitored (Fig. 1 b). For the sake of simplicity, we consider here the case of the pulling direction $\hat{\tau}$ in the plane of the ruler.

As the flap is pulled, a fold first forms, and the crack eventually propagates. We experimentally observe that the line joining the fracture head to the pulling point (after folding) is almost straight and is thus parallel to the pulling direction $\hat{\tau}$. Depending on the relative orientation of pulling (Fig. 1p), fracture propagates either at a departing angle $\theta$ (see Fig. 1, ) or along the ruler, $\theta=\alpha_{r}$ (as depicted in Fig. 11). (a)

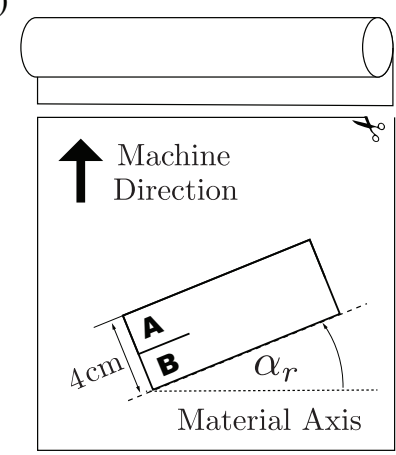

Fracture Direction

(c)

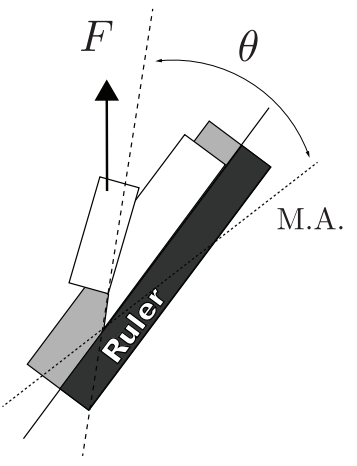

(b)

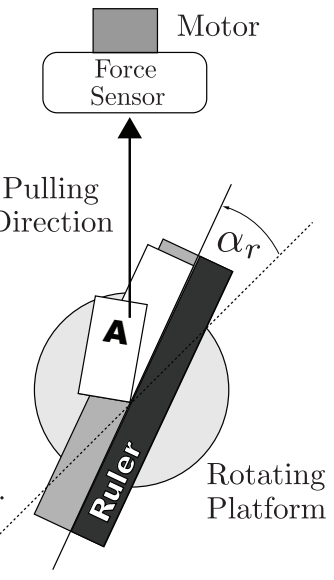

Ruler Axis

(d)

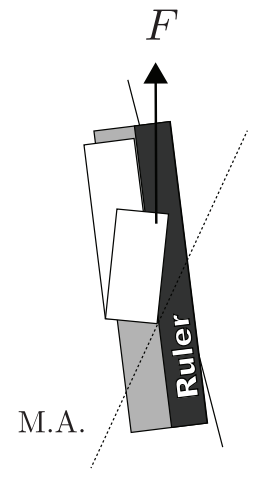

FIG. 1. Experimental setup. a) A rectangular specimen of specified dimensions is cut with an angle $\alpha_{r}$ with respect to the material axis. A cut is additionally made along its center line in order to separate two "legs". b) The specimen is maintained on an orientable platform by applying a ruler along its center line. The orientation of the ruler with respect to the material axis is given by the same angle $\alpha_{r}$. The resulting free leg is folded and pulled in the plane of the ruler following the "pulling direction" through a micro positioning system. The pulling force $F$ is monitored with a load cell. c) Typical case of a fracture propagating away from the ruler along a generic direction $\theta$ defined with respect to the material axis. d) Case of a fracture following the ruler $\left(\theta=\alpha_{r}\right)$.
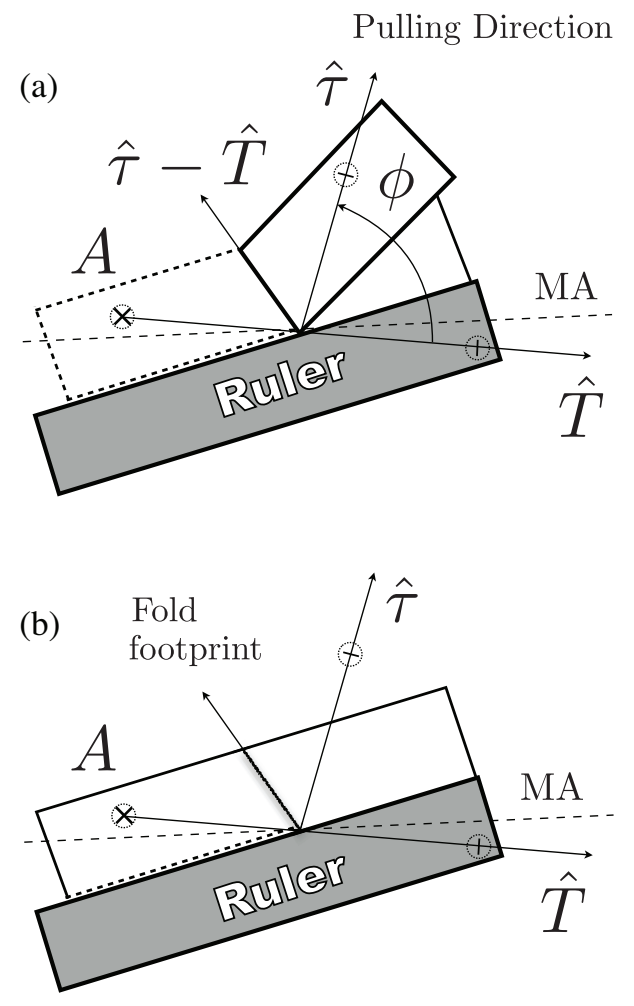

FIG. 2. Simplified geometry of the tearing experiment. a) Flap diagram indicating the pulling point prior flap folding (dashed line); the folded flap and the position of pulling point during pulling. The unit grip $\hat{T}$ and pulling $\hat{\tau}$ vectors are defined through the initial and the current positions of the pulling point and the fracture head. The direction of the fold corresponds to the perpendicular bisector, $\hat{\tau}-\hat{T}$. b) Geometry of the tearing on the flat sheet diagram. The location of the fold is indicated for clarity.

\section{THIN ISOTROPIC SHEET}

We first consider the case of thin sheets of negligible bending stiffness. It will prove useful to define at each time the "grip" unit vector $\hat{T}$, which joins the initial location of the pulling point to the fracture tip (Fig. 2a). Although the sheet is strongly bent in the experiments as in Fig $2 a$, the prediction for fracture propagation is best understood by considering the trajectory in the diagram in Fig. 2 $\mathrm{b}$, where the sheet is replaced in its flat configuration. Our theoretical approach is based on Griffith's criterion for fracture propagation which establishes that the energy release rate should exactly compensate the cost for creating new surfaces [22]. As the sheet is here assumed to be inextensible and with a vanishing bending stiffness, there is no elastic energy. As a consequence, we expect fracture propagation (in a sheet with thickness $h$ and fracture energy $G_{c}$ ) to occur over a distance $\mathrm{d} s$ when the cost of fracture $G_{c} h \mathrm{~d} s$ is balanced by the work of the external force $F \mathrm{~d} l$, where $\mathrm{d} l$ is the displacement of the 
pulling point $P$ in the pulling direction $\hat{\tau}$ (Fig 3 ). Two terms contribute to $\mathrm{d} l$ as the pulling point moves to from $P$ to $P^{\prime}$ and the crack tip from $O$ to $O^{\prime}$, respectively. The change of the length of the flap $O P$ to $O^{\prime} P^{\prime}$ is by construction equal to the change from $A O$ to $A O^{\prime}$ and thus writes $\mathrm{d} l_{\hat{T}}=\hat{T} \cdot \hat{t} \mathrm{~d} s$ (to the first order of $\mathrm{d} s$ ). In addition to this change in length, the crack tip moves along $\mathrm{d} s$. The contribution of this displacement in the direction $\hat{\tau}$ is given by: $\mathrm{d} l_{\hat{\tau}}=\hat{\tau} \cdot \hat{t} \mathrm{~d} s$. The global displacement of the pulling point in the direction $\hat{\tau}$ thus follows:

$$
\mathrm{d} l=\left(d l_{\hat{\tau}}+d l_{\hat{T}}\right)=(\hat{\tau}+\hat{T}) \cdot \hat{t} \mathrm{~d} s .
$$

Griffith's criterion for fracture propagation

$$
F d l=G_{c} h \mathrm{~d} s
$$

can therefore be rewritten as

$$
2 F \cos \frac{\phi}{2} \cos \left(\theta-\alpha_{t}\right)=G_{c}(\theta) h,
$$

where $\alpha_{t}$ is the orientation of vector $\hat{\tau}+\hat{T}$ with respect to the material axis and $\phi$ is the angle between vectors $\hat{\tau}$ and $\hat{T}$ as defined in Fig. 3 (since $\hat{\tau}$ and $\hat{T}$ are unit vectors, $\|\hat{\tau}+\hat{T}\|=2 \cos (\phi / 2))$. We can also express the energy release rate as $G(\theta)=2 F \cos (\phi / 2) \cos \left(\theta-\alpha_{t}\right) / h$, which is maximum for $\theta=\alpha_{t}$. In this isotropic material, fracture propagates in the direction that maximizes the energy release rate 22, or equivalently in the direction that minimizes the fracture force. We conclude that in an isotropic, infinitely bendable sheet, where the pulling point defining a grip vector $\hat{T}$ is pulled along direction $\hat{\tau}$,

$$
\text { fracture proceeds in the direction of vector } \hat{T}+\hat{\tau}
$$

ie, along the bisectrix of pulling and grip vectors $(\hat{\tau}, \hat{T})$. In the following, the vector $\hat{T}+\hat{\tau}$ will be referred to as "tearing vector". Note that, counter-intuitively, the fracture direction does not coincide with the pulling direction $\hat{\tau}$. Rather, it propagates in a direction perpendicular to the fold axis, a feature also observed in other cases of fracture in infinitely bendable sheets 23]. Indeed, as bending energy has been neglected, the fold is strongly focalized, leading to a ridge that is parallel to the direction of $\hat{\tau}-\hat{T}$, perpendicular to the tearing vector.

We now present the consequences of the geometrical rule (3) on the features of fracture guided by a ruler. Three cases can be distinguished namely, following the ruler for guided tearing, symmetric, and departing away from the ruler. For the discussion it is useful to define the angle $\alpha$, as the orientation of the ruler with respect the grip vector (Fig. 4 ).

a) Guided tearing: the tearing vector is oriented towards the ruler (Fig. 4 4 ). In this case, the presence of the ruler frustrates propagation along the natural tearing vector and the crack path follows the ruler. This is the situation of guided tearing that we use in everyday life when we wish to control the direction of the cut.

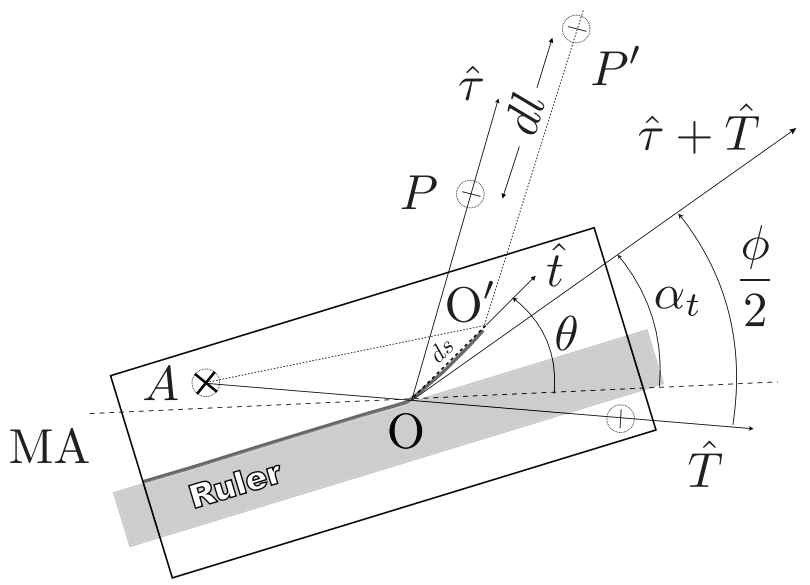

FIG. 3. Sketch depicting fracture progression. Fracture advances a distance $d s$ following a generic angle $\theta$. $d l$ is the total increment displacement of the pulling point, from $P$ to $P^{\prime}$, along the pulling vector $\hat{\tau}$.

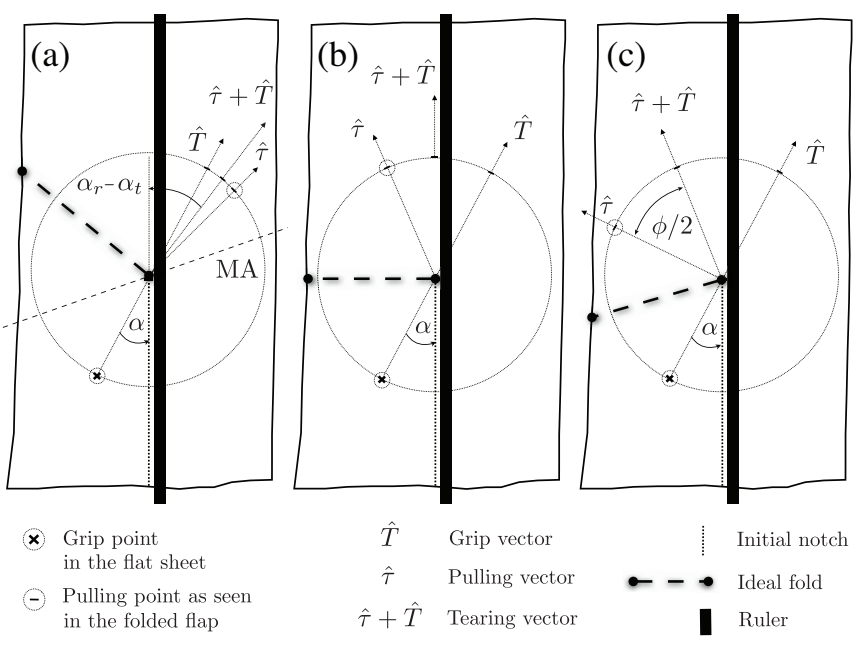

FIG. 4. Different ruler cases. a) The tearing vector is oriented towards the ruler and the crack path follows the ruler b) Symmetric case: the tearing vector, $\hat{T}+\hat{\tau}$, is parallel to the ruler; critical guiding. c) The tearing vector departs from the ruler.

For a successful control of the tearing, we must therefore make sure that the bisector of pulling and grip vectors $(\hat{\tau}, \hat{T})$ points inside the ruler. Counter-intuitively, this condition may be achieved even when pulling the sheet away from the ruler. If fold-ruler friction is minimized, guiding is possible for the tearing vector ranging from being parallel (dangerously close to loosing the control of the propagation departing the ruler) to perpendicular to the ruler (apparently safest situation). The latter case is achieved by orienting the pulling vector at an angle equal to $\pi-\alpha$ with respect to the ruler (i.e. the symmetric of grip vector with respect to perpendicular to the ruler), leading to a fold that is parallel to the ruler. However, 
the force applied by the operator in the general guided case is given through Eq. 2 as

$$
F=\frac{G_{c} h}{2 \cos (\phi / 2) \cos \left(\alpha_{r}-\alpha_{t}\right)}
$$

The tearing force would thus diverge when the tearing vector (with an angle $\alpha_{t}$ ) becomes perpendicular to the ruler, $\alpha_{r}-\alpha_{t}=\pi / 2$, and the fold is parallel to the ruler. Such large forces may trigger additional fracture in the sheet and are therefore not to be favored. The tearing force (4) is minimal when $\alpha_{r}=\alpha_{t}$, i.e. when the tearing vector lies parallel to the ruler, or equivalently when the grip and pulling vector are symmetric with respect to the ruler, a situation that we study next.

b) Symmetric tearing: the tearing vector is parallel to the ruler leading to a fold line perpendicular to the ruler (Fig. 4 4 ). The grip point may still be chosen anywhere in the quarter of the plane defined by the notch and the perpendicular to the ruler passing through the crack tip $(0<\alpha<\pi / 2)$. The limiting configuration occurs when the grip vector and pulling vector are both perpendicular to the ruler. In terms of the work of the operator, we deduce from Eq. (4) that the less costly configuration is $\phi=0(\alpha=0)$, for which $F=G_{c} h / 2$. We conclude that the optimal situation for guiding tearing is when picking the pulling point almost at the contact of the ruler, and the operator pulls it along the ruler. However this situation corresponds to the limit case where the crack is close to depart from the ruler.

c) Departing from the ruler: the tearing vector points away from the ruler edge (Fig. 4 4 ). Under this condition, fracture is not guided anymore and its propagation is dictated by the tearing vector, $\theta=\alpha_{t}$. Then, the force applied for the operator depends solely on the angle $\phi$ between grip and pulling vectors,

$$
F=\frac{G_{c} h}{2 \cos (\phi / 2)}
$$

\section{QUANTITATIVE EXPERIMENTAL TESTS}

In the following, we experimentally explore what geometrical features of the tearing problem are captured by the above analysis.

We first explore the dependence of the fracture path with the tearing vector $\hat{\tau}+\hat{T}$. We use here samples cut perpendicularly to the direction of the material axis $\left(\alpha_{r}=90^{\circ}\right)$. We select a pulling vector $\hat{\tau}$ oriented at $45^{\circ}$ departing the ruler and vary the angle $\alpha$ made by the grip vector $\hat{T}$ with the ruler (Fig. 5). The initial notch is made here parallel to the ruler at a distance of $5 \mathrm{~mm}$. This offset of the crack tip from the ruler allows us to probe the prediction (3) without hitting the ruler. Note that according to our experimental choices, the tearing vector will be parallel to the ruler, ie $\alpha_{t}=\alpha_{r}=90^{\circ}$, for $\alpha=45^{\circ}$. We thus expect the fracture path to depart from the ruler for $\alpha<45^{\circ}$ and to follow the direction given by the tearing vector $\alpha_{t}$. Conversely, we expect the fracture path to converge towards the ruler $\left(\theta \leq \alpha_{r}=90^{\circ}\right)$ for $\alpha>45^{\circ}$. Experiments confirm the theoretical predictions (Fig. 5) and we observe that even if the pulling vector points in a direction departing from the ruler (as is the case here), the fracture does propagates towards the ruler for sufficiently large $\alpha$. In these cases, we observe that once the fracture has reached the ruler edge, it proceeds along the ruler direction (black horizontal line Fig. 5).

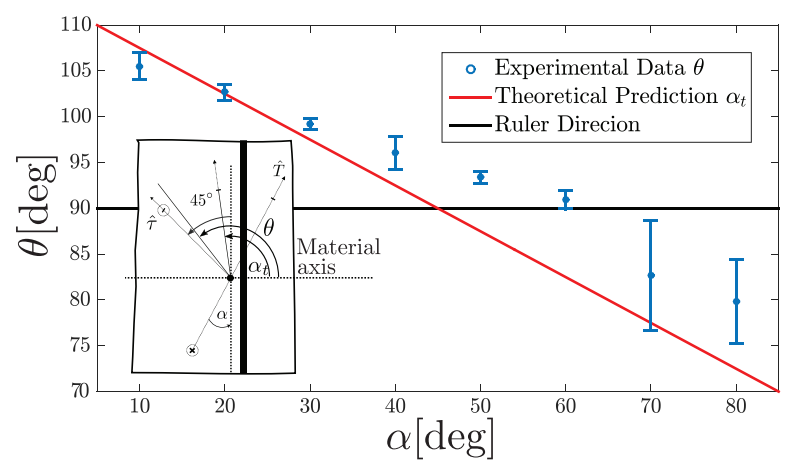

FIG. 5. Angle of fracture propagation $\theta$ as function of the position of the pulling point (orientation of grip vector $\alpha$ ) for a fixed pulling angle of $45^{\circ}$ with respect to the ruler. Red line indicates crack orientation as predicted by the tearing vector (3). Horizontal black line depicts the ruler orientation. Inset: Sketch of the geometry of the experiment.

We further test our model by fixing this time the pulling point, and varying the pulling direction. The grip vector $\hat{T}$ is selected parallel to the ruler and, with this choice $(\alpha=0)$, the angle of the pulling direction $\hat{\tau}$ with respect to the ruler is simply given by the pulling angle $\phi$. In particular, $\phi=0$ indicates the pulling direction that is exactly parallel to the ruler. We measure the fracture angle $\theta$ and the pulling force as a function of $\phi$ for two different orientations of the ruler with respect the Material Axis: oriented at $90^{\circ}$ and at $30^{\circ}$ (see insets in Figs. 6a and 6r, respectively).

Our simple analysis predicts that fracture propagates along the tearing angle $\alpha_{t}\left(\theta=\alpha_{t}=\phi / 2+\alpha_{r}\right)$, which corresponds to the solid blue line of slope 1/2 in Fig. 66(a, c). The presence of the ruler must then frustrate propagation angles beyond the ruler orientation $\alpha_{r}<\alpha_{t}$, that is when $\phi<0$, the propagation direction is $\theta=\alpha_{r}$ (blue horizontal line).

In a first experiment (Fig. 6a), with the ruler oriented along $90^{\circ}$, our data are in good agreement with the model, and fracture follows the ruler when its propagation along the tearing angle becomes frustrated (negative values of $\phi$ ). Nevertheless, in the second experiment where the ruler is oriented at $30^{\circ}$, an offset of about $6^{\circ}$ is 
systematically observed on the propagation angle $\theta$ with respect to $\alpha_{t}$ (Fig. 6r). For instance, for $\phi=0$, we observe that fracture propagates at an angle $\theta \approx 37^{\circ}$ which differs from the ruler angle $\left(\alpha_{r}=30^{\circ}\right)$. Fracture only becomes frustrated and guided by the ruler when the pulling vector is further oriented towards the ruler, with $\phi \lesssim-15^{\circ}$.

Eq. 2 accounts well for experimental values of the tearing force for the ruler oriented at $90^{\circ}$ in the regime where the fracture does not follow the ruler (the blue line in Fig. 6b). When the trajectory of the fracture is at the edge of the ruler, the fold is in contact with the ruler leading to noticeable frictional forces. In the case of guided cracks $\left(\theta=\alpha_{r}\right)$, Eq. 4 underestimates the pulling force by about thirty percent. When adding a Coulomb friction force of the form $F=(1+\mu) F_{c}$, a good agreement is obtained (green line in Fig. 6b), with $\mu \approx 0.3$ and $F_{c}$ given by Eq. 4. A similar effect of friction is observed when the ruler is oriented at $30^{\circ}$ (Fig. 6d). However, the tearing force deviates here significantly from the prediction from Eq. (2) even for non frustrated directions (blue line).

In conclusion, while our simple geometrical approach based on the assumption of material isotropy and negligible elastic energy captures the fundamental propagation features, we find that the fracture trajectory as well as the pulling force clearly depend on film orientation, revealing fracture-anisotropy or elastic-energy interplays. In the following section, we develop more refined arguments to predict fracture direction accounting for both the fracture energy anisotropy and the elastic energy stored in the fold.

\section{FRACTURE ANISOTROPY AND FOLD EFFECTS}

\section{Fracture Criterion in Anisotropic Materials}

Thin films are commonly anisotropic due to manufacturing procedures. Even for weak anisotropy, small variations of the fracture energy with orientation can affect the fracture trajectory significantly. We therefore recall the fracture criterion for anisotropic materials used recently in [1, 15. According to the classical Griffith criterion, a crack propagates in the generic direction $\theta$ for which the energy release per unit of fractured surface (the Energy Release Rate $G(\theta)$ ) compensates the energy cost of fracturing the material $G_{c}(\theta)$, so that $G(\theta)=G_{c}(\theta)$. This criterion alone does not predict the direction of propagation, and an additional criterion is needed. In an isotropic material, and for smooth propagation, it is assumed that fracture propagates in the direction that maximizes the Energy Release Rate 24]. This "Maximum Energy Release Rate Criterion" is equivalent to the Principle of Local Symmetry for continuous trajectories [18, 19]. In the case of an anisotropic material, a natural generaliza-
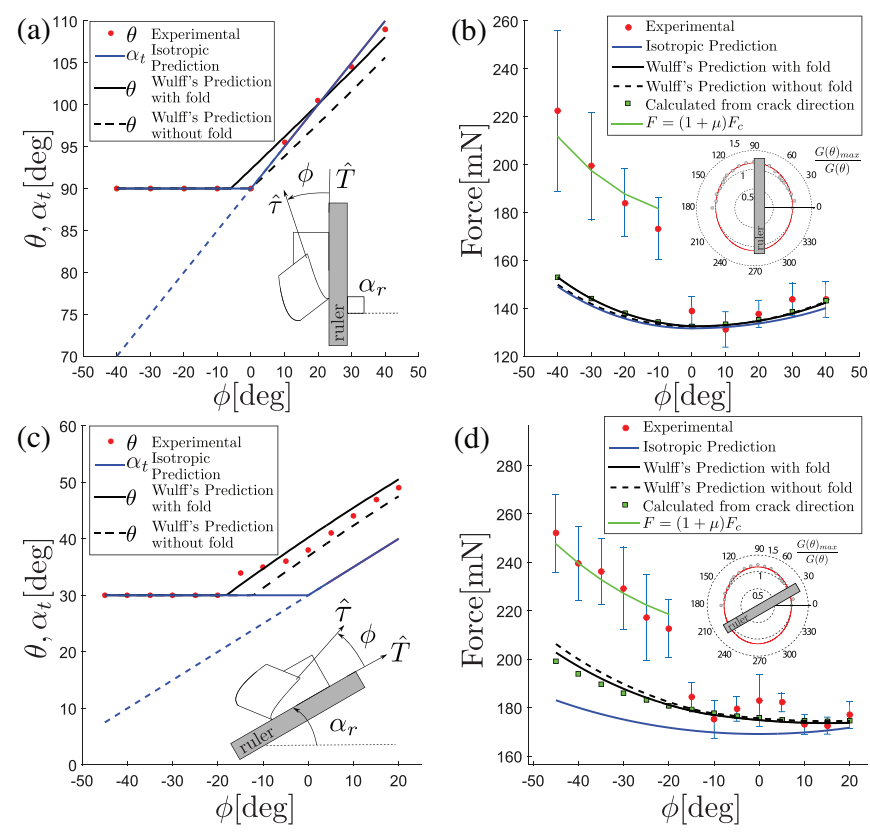

FIG. 6. Angle of fracture $\theta$ and tearing force $F$ as function of $\phi$ (measured with respect to the ruler). a) and b) ruler oriented at $90^{\circ}$ with respect to the film axis. c) and d) ruler oriented at $30^{\circ}$ with respect to the film axis. In a) and c) predictions from geometrical arguments, $\theta=\alpha_{t}$, (without ruler constrain) are represented by straight blue lines. For $\phi<0$, solid horizontal blue lines indicate propagation along the ruler, $\theta=\alpha_{r}$. Below these lines, no fracture propagation can occur due to the presence of the ruler. Dashed black lines are the predictions including anisotropy (Wulff's without fold), whereas solid lines include in addition the effect of the fold stiffness. In b) and d) geometrical model predictions for the tearing force from Eq. 2 are indicated with blue lines. When fracture is guided by the ruler, $\theta=\alpha_{r}$, the tearing force is given by Eq. 4 Dashed black lines indicate corrections due to fracture anisotropy (Wulff's plot) while solid black lines indicate predictions including both, anisotropy and fold effects. Insets in b) and d) indicate the inverse of fracture energy as a function of the propagation angle $\theta$, and the ruler orientation.

tion of the Maximum Energy Release Rate criterion is to postulate that fracture propagates in the first direction which satisfies Griffith's criterion [19, 25,27]. Thus, assuming that the loading is progressively increased, cracks propagate in direction $\theta$ which first satisfies

$$
G(\theta)=G_{c}(\theta)
$$

therefore imposing

$$
\frac{d G(\theta)}{d \theta}=\frac{d G_{c}(\theta)}{d \theta}
$$

As a result, crack propagation does not in general follow the direction of maximum energy release rate, but is attracted towards directions less costly in fracture energy. The condition (7) can be identified as an Eshelby torque 
(left hand side) balancing a material torque associated with anisotropy in fracture energy [26]. This general criterion (Eqs. 6 and 7) was tested in numerical phase field approach 25 27. Recently, the tearing path observed in anisotropic films pulled from two points 1, 15, was shown to obey this criterion. In addition, the direction of propagation of interacting cracks in thin films adhered to a rigid substrate has been described with the same approach, despite differences in geometry and underlying physics [28, 29].

\section{Measuring anisotropic fracture energy in a simplified geometry}

As in previous studies [1, 15, the bioriented polypropylene sheets used here are "balanced material" that exhibits weak anisotropy (Young's modulus with less than $20 \%$ variation around $1.8 \mathrm{GPa}$ ) due to bi-axial stretching during extrusion. We determine the fracture energy as a function of orientation using the trouser-test methodology as described in 1 and 15. Briefly, the sample is a rectangular strip (20 $\mathrm{mm}$ width and $100 \mathrm{~mm}$ long) with a longitudinal cut splitting the strip in two flaps with equal width. Both flaps are clamped and pulled symmetrically away from each other (see Figs. $7 \mathrm{a}$ and $7 \mathrm{~b}$ ). Samples are cut in several orientations $\alpha_{t}$ with respect to the material axis, and the pre-cut defines the initial position of the crack tip. As a result of symmetry in loading, the tearing vector is parallel to the side of the strip, along the orientation $\alpha_{t}$ (Fig. 7a). As the material is anisotropic, the fracture propagates in a direction $\theta \neq \alpha_{t}$, for a given applied force $F$, both of which are measured for each sample. The fracture energy $G_{c}$ for the orientation observed $\theta$ is then deduced from Griffith's criterion, which writes $G_{c}(\theta)=2 F \cos \left(\theta-\alpha_{t}\right) / h$ and is reported in Fig. 7b. The fracture energy is well approximated by the simple equation $G_{c}(\theta)=G_{1} \cos ^{2}(\theta)+G_{2} \sin ^{2}(\theta)$ (fig. 7 k). We observe that $\theta=\alpha_{t}$ (straight propagation) occurs in samples along the "Axis 1" (the material axis) and its perpendicular (see Fig. 7a), as one expect from the symmetry imposed in manufacturing. In this material, plastic dissipation results in a rather large fracture energy $G_{c} \sim 5 \mathrm{~kJ} / \mathrm{m}^{2}$, however, the process zone remains smaller than the thickness $(h=50 \mu \mathrm{m})$ [1].

\section{How anisotropy deflects fracture}

In the case of an isotropic material, the energy release rate is optimized when the fracture direction $\hat{t}$ is parallel to the tearing vector, $\hat{T}+\hat{\tau}$. In the case of anisotropic material, the direction of propagation is set by Eshelby's condition (Eq. 7), which reads here

$$
-2 F \cos \left(\frac{\phi}{2}\right) \sin \left(\theta-\alpha_{t}\right)=\frac{d G_{c}(\theta)}{d \theta} h .
$$

(a)

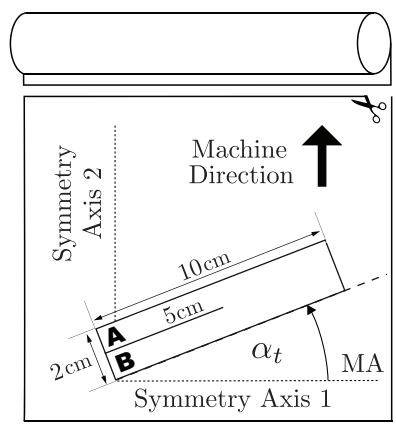

(b)

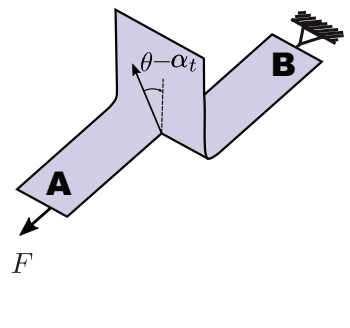

(c)


FIG. 7. a) The sample dimensions and its orientation with respect to the material axis (Axis-1) and the machine direction (Axis-2). b) Trouser test configuration for the measure of the fracture energy $G_{c}(\theta)$ as function of the fracture direction $\theta$. c) The Polar plot of the fracture energy as a function of $\theta$. The solid line is the best fit to the experimental data of the form $G_{c}(\theta)=G_{1} \cos ^{2}(\theta)+G_{2} \sin ^{2}(\theta)$. Values of $G_{1}=6.0 \mathrm{~kJ} / \mathrm{m}^{2}$ and $G_{2}=5.3 \mathrm{~kJ} / \mathrm{m}^{2}$. d) The Wulff's type diagram $G_{c}^{-1}(\theta)$ construction leads to find graphically the direction of propagation $\theta_{w}$, as the first intersection point (indicated by a red circle), and the force $F$ required for a given tearing direction $\alpha_{t}$.

Equations $(2)$ and $(8)$ then lead to

$$
\tan \left(\theta-\alpha_{t}\right)=-\frac{d G_{c}(\theta)}{d \theta} \frac{1}{G_{c}(\theta)},
$$

which, given the fracture energy $G_{c}(\theta)$ and the direction of tearing vector $\alpha_{t}$, allows for the determination of the propagation angle $\theta$. Note that if the material is isotropic, we recover propagation along the tearing vector since $\theta=\alpha_{t}$.

An equivalent and elegant way to determine the direction of propagation is to use a graphical construction analogous to Wullf's construction introduced in the context of anisotropic interface growth [1, 15. Here we apply this construction to the case of tearing with a ruler, where the tearing vector is oriented along an angle $\alpha_{t}$ (configuration considered in Fig. 6. We plot in polar coordinates $(r, \theta)$ the fracture energy curve $r(\theta)=1 / G_{c}(\theta)$, which we referred to as $\gamma^{-1}$-curve in [1]. We add the Energy Release Rate curve $r(\theta)=\left[2(F / h) \cos (\phi / 2) \cos \left(\theta-\alpha_{t}\right)\right]^{-1}$, which corresponds to a straight line oriented along direction $\alpha_{t}+\pi / 2$ at a distance $h / 2 F \cos (\phi / 2)$ from the origin 


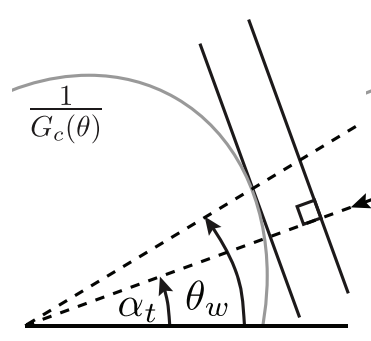

(a)

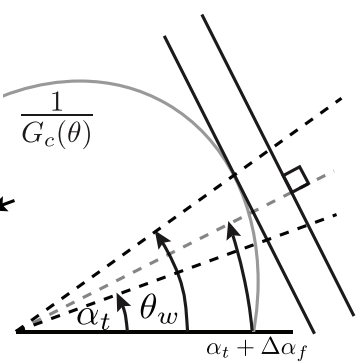

(b)
FIG. 8. a) Polar Wulff's diagram for anisotropic fracture energy. The $\gamma^{-1}$ curve: $1 / G_{c}(\theta)$ (gray line) and the straight lines defined by $\left[2(F / h) \cos (\phi / 2) \cos \left(\theta-\alpha_{t}\right)\right]^{-1}$ are plotted for two particular values of $F$ (black solid lines). Arrows on the dashed line indicate the direction of increase of the tearing force and $\theta_{w}$ denotes the propagation direction obtained at intersection. For the isotropic case, intersection occurs for $\theta_{w}=\alpha_{t}$. b) The generalized Wulff's diagram which considers both the tilt angle $\Delta \alpha_{f}$ induced by the fold and the anisotropy of the fracture energy. An effective tearing direction can be defined as $\alpha_{t}+\Delta \alpha_{f}$.

( Fig. 8p). As $F$ is progressively increased, this line comes closer to the origin but keeps its initial orientation. Griffith criterion (Eq. 2) is satisfied at any intersection point of the line with the $\gamma^{-1}$-curve and is therefore is first satisfied at the first point of intersection. According to the minimization criterion, the tearing force, and the direction of propagation $\theta$ are therefore defined by the point of the $\gamma^{-1}$-curve which accepts a tangent along direction $\alpha_{t}+\pi / 2$. This criterion was successfully tested [1] in the simpler symmetric "trouser" geometry.

However, when we apply this anisotropic construction to tearing experiments with ruler, the predictions (black dashed lines in Fig. 6(a, c)) exhibit discrepancies when compared to the experimental fracture angles. Although accounting for material anisotropy improves the prediction of the direction of propagation when the ruler is oriented at $\alpha_{r}=30^{\circ}$ (Fig. 6c), significant mismatch is observed with other orientations (see Fig. 6a, for a configuration with the ruler at $\alpha_{r}=90^{\circ}$ ). This observation suggests that an additional ingredient is at play in the selection of the direction of crack propagation.

\section{How bending energy deflects fracture}

We have up to now neglected the elastic energy of the sheet, which is localized in the region of the fold. This bending energy varies during fracture propagation due to the geometric variations of the fold and may contribute to the energy release rate. Accounting for the variation of elastic bending energy in Griffiths's criterion leads to:

$$
G_{c}(\theta)+\frac{d U_{f}}{d w} \frac{d w}{d s}=F \frac{d l}{d s},
$$

where $U_{f}$ is the elastic energy stored in the fold and $w$ the width of the fold (proportional to the width of the flap). In order to obtain an estimate of $\frac{d U_{f}}{d w}$, we assume a nearly conical shape for the fold, which is supported by direct measurements of fold shape. Within this assumption, the local radius at the position $z$ along the fold is given by $r=r_{c}+\frac{r_{w}-r_{c}}{w} z$, where $r_{c}$ and $r_{w}$ are the radii of curvature of the fold at the fracture tip and at the free end, respectively (see Fig. 9). We obtain for the bending energy,

$$
U_{f}=\int_{0}^{w} \frac{B}{2 r^{2}} \pi r \mathrm{~d} z=\frac{\pi}{2} \frac{B w}{r_{w}-r_{c}} \ln \frac{r_{w}}{r_{c}}
$$

where $B=E h^{3} / 12\left(1-\nu^{2}\right)$ is the bending stiffness of the sheet of thickness $h$, Young modulus $E$ and Poisson coefficient $\nu$.

Since $\mathrm{d} l / \mathrm{d} s=(\hat{T}+\hat{\tau}) \cdot \hat{t}=2 \cos (\phi / 2) \cos \left(\theta-\alpha_{t}\right)$ and $\mathrm{d} w / \mathrm{d} s=-\sin \left(\theta-\alpha_{t}\right)$, Eq. 10 reads:

$G_{c}(\theta)-\frac{\pi}{2} \frac{B}{r_{w}-r_{c}} \sin \left(\theta-\alpha_{t}\right) \ln \frac{r_{c}}{r_{w}}=2 F \cos \frac{\phi}{2} \cos \left(\theta-\alpha_{t}\right)$

The balance of the moment induced by the component the force perpendicular to the fold $F \cos (\phi / 2)$ and the moment at the base of the fold leads to:

$$
F \cos (\phi / 2)=\int_{0}^{w} \frac{B}{2 r^{2}} \mathrm{~d} z=\frac{B w}{2 r_{c} r_{w}}
$$

We finally obtain a minor correction for the effective fracture energy:

$$
G_{c}(\theta) \simeq 2 F \cos \phi / 2 \cos \left(\theta-\left[\alpha_{t}+\Delta \alpha_{f}\right]\right)
$$

with

$$
\Delta \alpha_{f}=\pi \frac{r_{c} r_{w}}{\left(r_{w}-r_{c}\right) w} \ln \frac{r_{w}}{r_{c}} \ll 1 .
$$

In our experiment, $r_{c} \simeq 0.5 \mathrm{~mm}, r_{w} \simeq 6.5 \mathrm{~mm}, w \simeq$ $20 \mathrm{~mm}$, which leads to $\Delta \alpha_{f} \approx 10^{\circ}$. When comparing Eq. (11) with Eq. (2), we see that the effect of bending energy in our simple model is simply to replace $\alpha_{t}$ by $\alpha_{t}+\Delta \alpha_{f}$, which corresponds to an effective rotation of the tearing vector by an angle $\Delta \alpha_{f}$. In the absence of anisotropy, we expect fracture propagation along the direction $\left[\alpha_{t}+\Delta \alpha_{f}\right]$. The bending energy of the fold thus tends to deflect the propagation towards the most bent flap, which is also observed in other situations [2. Implementing the effect of bending energy in our graphical prediction of the direction of the crack propagation results in a simple rotation of the solid lines by an angle $\Delta \alpha_{f}$, as shown in 8 b.

The predictions of this modified geometrical construction are reported in Fig. 6 in black continuous line for 


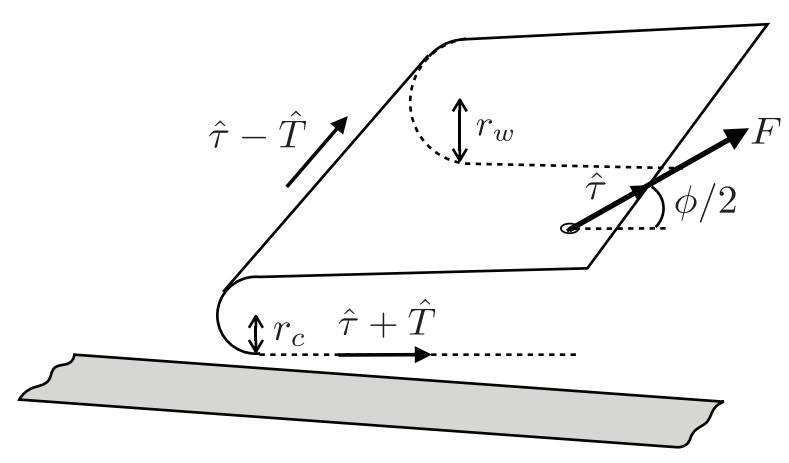

FIG. 9. Geometry of the fold as bending energy is taken into account. The fold is assumed to have a conical shape, with a radii of curvature $r_{c}$ and $r_{w}$ at the fracture tip and at the free end, respectively.

a value of $\Delta \alpha_{f}=6^{\circ}$, which results in a better comparison with experimental data (Fig. 6(a,c)). Notice that neglecting both the fold energy and the fracture energy anisotropy (isotropic prediction) leads to good matching to the experimental data in Fig. 6a. This is due to a fact that under the conditions of Fig. 6a, anisotropic effects are reduced - small axis of symmetry along the ruler, fracture is attracted to the ruler - and fold energy produce corrections in the propagation angle that tend to compensate anisotropic effects, repelling fracture from the ruler.

Although the bending energy stored in the fold plays an important role in determining fracture direction, it does not affect significantly the pulling force in our experiment where anisotropy remains small $\left(\left(G_{1}-G_{2}\right) / G_{1} \sim 11 \%\right)$. Indeed, when $\alpha_{t}$ is close to 0 or $\pi / 2$, where the fracture energy is respectively maximal or minimal, $\Delta \alpha_{f}$ locally varies only at second order with orientation, leading to a minor change in tearing force. The effect of the fold is more significative along the orientation of maximum anisotropy (where $d G / d \theta$ is larger) but still remains modest (see Fig 6d). Finally, when the fracture proceeds along the ruler, the force significantly deviates from Wulff's predictions since friction becomes important.

\section{CONCLUSION}

We have investigated the familiar tearing of a sheet along a ruler. In contrast to the classical trouser test where two symmetric folds are at play, the presence of the ruler imposes boundary conditions on one of the flaps as it forbids its bending. As a consequence, the curvature of the fold does affect the crack trajectory. In our experiment, we start by picking an arbitrary point on the flap which will be pulled. The direction joining this point to the crack tip defines the unit grip vector $\hat{T}$. This point is then pulled along a vector $\hat{\tau}$. We demonstrated that the geometry of the configuration determines the main features of fracture through a tearing vector $\hat{\tau}+\hat{T}$.

Indeed, in the case of an isotropic, infinitely thin sheet, fracture direction occurs along this tearing vector, and propagation is guided by the ruler when fracture hits it, which eventually occurs when the tearing vector is oriented towards the part of the sheet covered by the ruler. Counter-intuitively, this guided configuration does not require the pulling direction to be oriented towards the ruler. In terms of lowest pulling force, the optimal configuration corresponds to a pulling point placed initially very close to the ruler and pulled parallel to the ruler. Nevertheless, this ideal direction of propagation is in practice very sensitive to additional effects that we have identified.

First, fracture energy anisotropy can lead to significant deviation of fracture direction. Fracture is indeed deflected towards direction of the minimum of fracture energy. We quantify this effect using a geometrical solution similar to Wulff's construction, an elegant tool from cristal growth. Second, finite bending energy in the fold leads to an additional shift in the orientation of the fracture. Through a simple model accounting for the fold energy, we show that this feature can be captured by means of the generalization of Wulff's construction. We find that the fold always favors propagation departing the ruler (since fracture tends to release the fold energy by decreasing its width). Conversely, anisotropy of fracture energy may favor propagation towards or departing the ruler, as it induces a shift towards the direction of minimal fracture energy.

We conclude that efficient control of fracture by a ruler is attained when fracture is constantly directed towards the ruler, making it insensitive to imperfections. This is achieved by either a pulling point initially not too close to the ruler, or by a pulling direction oriented more towards the ruler than necessary, which involves a higher tearing force than the optimal solution with in addition a supplementary component due to friction along the ruler.

\section{Acknowledgments}

F.M. acknowledges ANID-Chile through Fondecyt project N $\mathrm{N}^{\circ} 1201013$ and Fondequip 130149. Support from LIA-MSD France-Chile (Laboratoire International Associé CNRS, "Matière: Structure et Dynamique") is greatly acknowledged. We acknowledge the support from DICYT Grant USA2055-042031MH-POSTDOC of Universidad de Santiago de Chile. 
[1] A. Takei, B. Roman, J. Bico, E. Hamm, and F. Melo, Phys. Rev. Lett. 110, 144301 (2013).

[2] E. Hamm, P. Reis, M. LeBlanc, B. Roman, and E. Cerda, Nat. Mater. 7, 386-390 (2008).

[3] E. Bayart, A. Boudaoud, and M. Adda-Bedia, Phys. Rev. Lett. 106, 194301 (2011).

[4] F. Brau, Phys. Rev. E 90, 062406 (2014).

[5] Y. Mai and B. Cotterell, Int. J. Fracture 24, 229-236 (1984).

[6] C. Muscat-Fenech and A. Atkins, Fatigue Fract. Eng. Mater. Struct. 17, 133-143 (1994).

[7] E. Sharon, B. Roman, M. Marder, G.-S Shin, and H. Swinney, Nature 419, 579 (2002).

[8] V. Romero, B. Roman, E. Hamm and E. Cerda, Soft Matter 9, 8282-8288 (2013).

[9] J.-F. Fuentealba, E. Hamm, and B. Roman, Phys. Rev. Lett. 116, 165501 (2016).

[10] B. Roman, P. Reis, B. Audoly, S. D. Villiers, V. Viguié, and D. Vallet, Comptes Rendus Mécanique 331, 811-816 (2003).

[11] A. Atkins, Eng. Fract. Mech. 74, 1018-1025 (2007).

[12] A. Ghatak and L. Mahadevan, Phys. Rev. Lett. 91, 215507 (2003).

[13] P. Reis, A. Kumar, M. D. Shattuck and B. Roman, Europhys. Lett. 82, 64002 (2008).

[14] O. Kruglova, F. Brau, D. Villers and P. Damman, Phys. Rev. Lett. 107, 164303 (2011).

[15] A. Ibarra, B. Roman, and F. Melo, Soft Matter 12, 59795985 (2016).

[16] A. Ibarra, J. F. Fuentealba, B. Roman, and F. Melo, Phys. Rev. E 100, 023002 (2019).
[17] B. Li, D. Millán, A. Torres-Sanchéz, B. Roman and M. Arroyo, J. Mech. Phys. Solids 119, 334-348 (2018).

[18] M. Amestoy and J.B. Leblond, Int. J. Solids Struct. 29, 465-501 (1992).

[19] A. Chambolle, G. A. Francfort, and J.-J. Marigo, J. Mech. Phys. Solids 57, 1614-1622 (2009).

[20] A. Atkins, Proc. 10th Congress on Materials Testing 3, 595 (1991).

[21] R. O'Keefe, Am. J. Phys. 62, 299-305 (1994).

[22] B. Lawn, Fracture of Brittle Solids, Cambridge University Press, 1993.

[23] B. Roman, Int. J. Fract. 182, 209-237 (2013).

[24] Another criterion proposed recently [30] is based on examination of the strain energy at the scale of the crack tip. Fracture is shown to propagate in the direction where this energy density is maximal. It remains to be shown how this microscopic criterion, introduced for inhomogeneous but isotropic material, is equivalent to the Maximum Energy Release Rate, and how it can be generalized to anisotropic materials.

[25] V. Hakim and A. Karma, Phys. Rev. Lett. 95, 235501 (2005).

[26] V. Hakim and A. Karma, J. Mech. Phys. Solids 57, 342368 (2009).

[27] B. Li, C. Peco, D. Millán, I. Arias, and M. Arroyo, Int. J. Num. Meth. Eng. 102, 711-727 (2015).

[28] J. Marthelot, B. Roman, J. Bico, J. Teisseire, D. Dalmas, and F. Melo, Phys. Rev. Lett. 113, 085502 (2014).

[29] J.-F. Fuentealba, J. Marthelot, B. Roman, and F. Melo, Phys. Rev. Lett. 124, 174102 (2020).

[30] L. Rozen-Levy, M. Kolinski, G. Cohen, and J. Fineberg, Phys. Rev. Lett. 125, 175501 (2020). 